\section{POLYCYSTIC OVARIAN SYNDROME}

\author{
Mishra S. ${ }^{*}$ Shrestha $A^{* *}$
}

\section{Address for Correspondence:-}

* Prof. Dr .S. Mishra Professor \& Head of the Department (Obstetrics \&Gynaecology)

U.C.M.S. Teaching Hospital, Ranigaon, Bhairahawa, Nepal

${ }^{* *} \mathrm{MD}$ (final year) Obstetrics \& Gynaecology Polycystic ovarian syndrome (PCOS) is an endocrine disorder that affects the reproductive system and is considered one of the leading cause of infertility. Nearly $10 \%$ of women worldwide of reproductive age group suffer from this.

In pcos, ovaries do not produce sufficient quantities of hormone required for follicles to mature. As a result follicles remain in the ovary and eventually turn into the cysts. These cysts accumulate in the ovary.

The menstrual clock depends on the interplay of several hormones that need to be produced in the right order. The FSH released by the pituitary gland helps to ripen one of the millions of follicles in a woman ovary every month.

The LH causes the follicle to rupture and release an ovum which is picked by the fallopian tube.

A third hormone called progesterone produced by the discarded shell of the follicle in the uterus plays a critical role in initiating the menstrual flow.

In women with pcos there is imbalance between FSH which stimulates the ovary to develop an egg, and LH which triggers the ovary to release the egg.The egg ripens but does not rupture. Instead egg starts accumulating in the ovaries. The LH levels are high by virtue of which the egg may start to grow and accumulate fluid but does not grow to mature enough to be released by breaking the follicle. Instead they remain as cysts in the ovary as a result ovulation does not occur. Unruptured follicles start producing male hormone such as testosterone which causes acne, male pattern baldness, and obesity and insulin resistance. Insulin resistance is the body's inability to absorb insulin and use it to break down carbohydrates. Even though insulin levels are normal the tissues do not respond to the insulin. Whatever carbohydrates are consumed gets converted into fat which leads to obesity. Women with pcos find hard to lose weight easily which further aggravates their insulin resistance. If this insulin resistance is not dealt with, pancreas may not be able to produce sufficient insulin to keep sugar levels in check leading to type2 diabetes. So women with pcos have increased risk of developing diabetes mellitus, cardiovascular diseases or hormone associated cancers including breast and endometrial cancer. 
It is believed to have a genetic component to pcos and increased chance of familial clustering of disease.

Long working hours, poor eating habits, stress and consumption of high fat animals which produce high arachidonic acid may contribute to this disease.

\section{SIGNS AND SYMPTOMS}

1. Infrequent menstrual periods or no periods

2. Infertility

3. Acne, oily skin

4. Weight gain and obesity

5. Male pattern baldness

6. increased growth of hair on the face,chest, and lower abdomen

7. Patches of thickened and dark brown or black skin on the neck, arms, breast or thigh.

8. HighBP

9. Pelvic pain

\section{DIAGNOSIS}

Medical history and complete physical examination is carried out

Height and weight to check obesity, bloodpressure, blood for hormone and glucose levels

Pelvic examination and ultrasound

\section{TREATMENT}

\section{For women not trying to conceive}

Birth control pills are an effective way of treatment, pills regularize menstrualcycle, reduces male hormone level, clear acne and other symptoms

\section{For women who want to conceive}

1. Fertility drugs like clomiphen and gonadotrophin which stimulates ovulation

2. Insulin resistance drugs like metformin are prescribed to stimulate ovulation

3. Drugs with combination of cyproterone acetate and ethinylestradiol. Cyproterone is an antiandrogenic and progestogenic while ethinylestradiol is estrogenic in action. This combination normalises hormonal ratio of $\mathrm{LH} / \mathrm{FSH}$, increase sex hormone binding globulin (SHBG) produces stable cycle control and improve ovulation when combined with metformin it helps reduce acne,hirsutism and androgenic alopecia.

4. Surgery-surgical removal of ovarian cysts by laparoscopy or open surgery restores hormone balance and regulates the ovarian cycle temporarily.

5. Ovarian drilling (PCO)-polycystic ovary, the presence of 12 or more follicles measuring $2-9 \mathrm{~mm}$ in diameters and increased ovarian volume greater than $10 \mathrm{cms}$. Ovarian drilling-ovary is punctured with a laser or an electrosurgical needle laproscopically which lowers the androgenic level and helps ovulation. Since PCOS is a metabolic disorder it cannot be cured, however it can be managed and even completely reversed by life style change with a proper balanced diet, a regular 
exercise programme and relaxation exercise like yoga.

\section{REFERENCES}

1.Baleri A.H. Conway GS KALTAS Get al Polycystic Ovarian Syndrome, The spectrum of disorder on 1741 patients.

2.Adams J, Polson DW, Franks S Polycystic Ovaries in women with anovulation and idiopathic hirustism BMJ Chin Res Edn L980 253355394.

3.Dahlgren E Johansson S., hindsjedt Get al Women with polycystic Ovary Syndrome Fertil Steril 155257505513.

4.Palep Singh M Mook. K Barth JH Balen AH An observation study in management and pelvic Ovarian Syndrome J Fam Plan Regional healthcare Zoon 30162165. 